\title{
Prof. Hong Jian: dreams come true-stay true to yourself!
}

Submitted Sep 24, 2017. Accepted for publication Sep 26, 2017.

doi: $10.21037 /$ jtd.2017.09.136

View this article at: http://dx.doi.org/10.21037/jtd.2017.09.136

\section{Introduction}

The ancient Greek aphorism "know thyself" engraved on the forecourt of the Temple of Apollo epitomizes the human desire for the exploration into the unknown. In fact, never has mankind dragged their feet in understanding themselves, as in the achievements scientists have made all these years in the studies of disease and pathology.

Ere the 2017 WCLC journey, AME Editors were sent across China to conduct in-depth interviews with an army of distinguished experts in the field of lung cancer. Together we learned how these soldiers fought for the nation that has the largest number of patients, and how they dedicated their lives to inventing the most-advanced weapons and probing into the enemy camp. From the past, they reflect and learn from mistakes; at present, they work hard and make changes; for the future, they make plans and improvements.

May this issue take you to the innermost world of these Chinese scholars, where you can delve into their wealth of knowledge and be inspired.

\section{Expert's introduction}

Hong Jian, a Chief Physician of Lung Cancer Clinical Medicine Center at Shanghai Chest Hospital, a master postgraduate advisor. Prof. Jian is a Committee Member of China Society of Oncology, and Department of Thoracoscopy, Shanghai Medical Association. She is also a Standing Committee member of Lung Cancer Molecular Targeting and Immunotherapy Professional Committee, Shanghai Anti-Cancer Association and a member of Shanghai Science and Technology Expert Library. In 1987, Prof. Jian graduated from Shanghai Second Medical University, and obtained a postgraduate degree. In 2008, she pursued professional studies in Chest Oncology at The University of California, San Francisco (UCSF), the United States.

Since the 90's, Prof. Jian has been studying small cells lung cancer for high-dose chemotherapy supported by hematopoietic stem cells, and won a dissertation award for Excellence, in Chinese Lung Cancer Research and a Third Prize of Shanghai Clinical Medicine Success.
After 2000, Prof. Jian first started the study of non-small cell lung cancer targeted therapy in Shanghai. She is mainly responsible for and participate a number of clinical researches in multi-centers in a national and international context on lung cancer epidermal growth factor and vascular growth factor for targeted therapy. Under an inspection, favorable criticisms were well received by Food and Drug Administration in the U.S. and European Union. In recent years, Prof. Jian started carrying out a research on the diagnosis of lung nodules and treating strategy, and won the First Prize of China Lung Cancer Conference for her best dissertation award in 2013. She participated in different theme-focused studies such as the national "Eight-Five Research" theme, "Thoracic Oncology", "Mountaineering Scheme" in Shanghai City and so on. Prof. Jian has currently finished two theme-focused topics in bureau level 2 and one theme-focused topic in civil service bureau level 1 as the First Leader in Charge. She has co-authored a number of books including Small Nodular Lung Cancer, Lung Cancer, Thoracic Oncology, Lung Cancer Chemotherapy and Targeted Therapy and 100 Questions of Lung Cancer, and published core journals for more than 10 articles both at home and abroad.

From 1987 onwards, Prof. Jian began to work on the diagnosis and treatment for respiratory diseases, and developed a solid clinical basis for respiratory diseases. Since the mid-90's, she mainly engaged in clinical diagnosis and treatment of lung cancer. She is good at diagnosing chest wall tumors, pleural mesothelioma, lung cancer, mediastinal tumors and other complicated diseases. Meanwhile, she has extensive clinical experiences in clinical chemotherapy of chest wall tumors, targeted therapy, immunotherapy and multidisciplinary comprehensive treatment.

"We are a lucky generation. From the era of national reform to the restoration of National College Entrance Examination, we bave had the opportunity to choose freely upon our own will. As I was conceiving my dream of becoming a 'white angel, I knocked on the door to the Medical School." said Prof. Jian (Figure 1). Deeply affected by films and novels, Prof. Jian has 


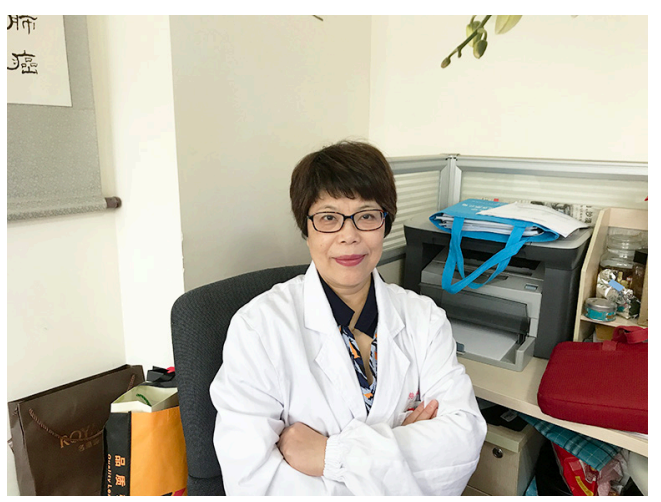

Figure 1 Prof. Hong Jian.

developed a strong passion for the "white gowns", which symbolized a doctor by profession. At this turning point in life, the seeds of dream inside her have begun to sprout, and she was then motivated to make the very first step into the medical world.

Thirty years flown by, Prof. Jian lamented at the hardship of being a doctor. She would have not chosen to become a doctor if she had known how exhausting work could have been in the first place. Nonetheless, after tasting the sweetness and bitterness, today she is more in love with being a doctor. From viewing her identity of being a doctor as a "career" to treating it as a "profession", Prof. Jian fondly deemed that this changed thought is mainly due to the nurturing and nourishing effects of medicine on an individual. Being a doctor is considered as a very professional career, not only does it require continuous learning on new knowledge, but it is also considered as a challenging career. In recent years, new knowledge requires rigorous thinking on treating diseases. Hence, doctors need to be well prepared mentally for various challenges. "Being in the medical field, we grow together with the industry, we must then follow and grow along with it." said Prof. Jian. Speaking of doctors that are facing enormous pressures on daily practices and scientific research, especially for those who entered the College during the 1980s, like Prof. Jian, and did not receive serious trainings of reaches, their scientific research works were made even more challenging. Despite whining at the challenges that met in work sometimes, Prof. Jian never thought about giving it up. As long as thinking about how she can help people out with their problems, her passion of work is reignited vigorously, fulfilling with her own life value by never giving up. Doctors started working from performing clinical practices to devising different clinical themes based on the typical mindset of solving clinical problems. By converting their clinical experiences to the results of scientific research, more clinical problems could be solved for patients, and doctors are able to fulfil their own life values from it, they are tired, complaining, yet feeling blessed and proud of themselves! I wonder, "Stay True to Yourself: Moving Forward" shall be the most descriptive interpretation for Prof. Jian work, which has best captured the core value of her career.

Experienced in thoracic surgery, Prof. Jian strongly believed "It is meaningless to have morals without skilled practices, while practicing without morals is very dangerous." As a doctor, we should pay fully respect to the basis of science, put ourselves in patients' shoes, and set the most suitable medical treatment for them based on their body conditions.

Tumors are manifold and complex. Prof. Jian encouraged young doctors to read more, learn more and think more. Just because we focused the study on lung cancer, it doesn't mean that we can view the study of other less common diseases in lungs as unimportant. On the contrary, by getting familiar with the basis of respiratory diseases that are related to lung cancer, it helps with a more precise diagnosis on whether who is with lung cancer and who's not. In terms of lung cancer, doctors are required to have the knowledge base of respiratory diseases, molecular biology, oncology, immunology, radiography and surgery. Hence, it helps with a more precise diagnosis and better at prolonging a patient's life under the approach of comprehensive diagnosis.

\section{Lung cancer: the high-risk groups and early diagnosis}

As everyone knows, smoking is the leading cause of Lung Cancer in modern society. Meanwhile, a clinical research found that for those who are over 40 years old, and usually have tiny nodules in which were swollen, are considered to be a high-risk group for lung cancer. For men, they usually smoked for more than 400 cigarettes a year. A recent clinical study has mentioned on a group of non-smoking women, who usually have a family history of cancer, is also considered to be a potential high-risk group for lung cancer. Prof. Jian suggested that for those who are over forties, having a smoking history or a family history of cancer, should go for a low dose of CT scan once a year. The early the detection of the cancer, the early the treatment one could be received. We should also be highly alert to the situation where many people are found smoking and have irritating dry cough, hoarseness, coughing out bloody 
sputum persistently, back pain, and swollen foots and hands. They are advised to go to hospital as soon as possible and do a low dose of CT scan.

Low-dose of CT scan can be used for early screening of lung cancer. Once there are any suspicious tumor lesions being found in the lungs, further examination could be done based on the places and sizes of lesions. For instance, a further examination is to be done for the lesions that lied in the hilum of the lung or lied near the area of the hilum of lung through a bronchoscopy or a tracheal biopsy. For the peripheral pulmonary solid nodules, which have more than $1 \mathrm{~cm}$ of diameter, a lung puncture could be performed. Pure ground glass nodules or part of the solid ground glass nodules will enhance the level of difficulty for performing a lung puncture. Therefore, it is needed to understand the relationship of its morphology, density, structure and adjacent tissues by carrying out the three-dimensional reconstruction of the lesion. For patients who did not have a clear tumor base, could be treated under the strategy of a clinical follow-up. Positron emission tomographycomputed tomography (PET-CT) is also considered as an effective means to detect tumors, yet it is less sensitive for detecting pulmonary nodules in which contains less than $10 \mathrm{~mm}$ in size.

\section{Ground-glass nodules: surgical resection vs. follow-up}

As the low-dose spiral CT examination of the chest is popular, small nodules are one of the most important manifestations of early stage lung cancer. About $10 \%$ of the population would be found pulmonary nodules during their first time trial of low-spiral CT scan. Studies reported that about $90 \%$ of the small nodules are benign lesions. Prof. Jian emphasized that after locating those small nodules, the first step is to perform a three-dimensional reconstruction of CT screening, then identify whether which of the pulmonary nodules are belong to pure ground glass nodules, partly solid glass nodules and solid nodules. In order to carry out an appropriate means of intervention to treat the patients properly, it is important for the doctors to be well aware of the size, shape (regular or not), border (clear or not) of the nodules, which will need further checking if there are any branches or if they are accompanied by pleural indentation (PI), as well as whether there are small burrs found in the surroundings, bubbles found in the middle area and so on. Meanwhile, a clinical follow-up is a crucial means of intervention on treating patients. Doctors should set up different follow-up approaches based on the diameters of the nodules, as some of the patients with small nodules might not appear with the related symptoms.

\section{Targeted therapy: mechanism \& applicable population}

When a particular gene mutation is detected in patients with non-small cell lung cancer, for genes such as epidermal growth factor receptor (EGFR), anaplastic lymphoma kinase (ALK) and receptor tyrosine kinase (ROS1) in cells, the signal transduction in cells changes, which results in an infinite number of cells' proliferation and eventually a tumor formation. A specific gene mutation is like a specific target, it makes use of a small molecule of drugs, and integrates with it together in order to block the signal transduction in the cell, so that an infinite proliferation of tumor cells is to be contained effectively. Nonetheless, not all of the patients or rich patients with advanced non-small cell lung cancer are suitable to receive targeted therapy. In fact, it depends on whether there is a specific gene mutation detected in them. Although the current targeted therapy has made new progress, it is still confined to several genes, such as EGFR, ALK, ROS1 and so on. Therefore, targeted therapy is appropriate for patients with therapeutic targets. It is of great importance to notice before the treatment or patients found relapsing after the treatment if there are any changes in the pathologies and genes of tumor biopsy. For patients with no specific gene mutation, Prof. Jian advised them to receive traditional chemical treatment.

Apart from adopting the living tissues from gene detection, fresh blood specimens are also available for gene detection. For advanced lung cancer patients, their DNAs have an amount of free tumor cells, the corresponding mutated targets can be detected through next generation sequencing. Then, doctors could be able to prescribe medication to patients clinically. If the patients' body conditions are not allowed to take the blood test and the blood test has a negative result at the same time, Prof. Jian advised these patients to adopt chemotherapy instead as a preferred choice.

\section{Lung cancer targeted therapy: the past, present and future}

In early 2000, lung cancer targeted therapy is applied in Chinese clinical practices. For over a decade, from the very 
beginning when we did not know if targeted therapy will be suitable for which types of patients to later on when we were entering the era of third-generation targeted chemotherapy, China has clearly achieved a huge success in targeted drug therapy for non-small cell lung cancer. Currently, the third-generation EGFR-TKI is applied in clinical practices, especially for the patients who had resisted the first- generation TKI and appeared with T790M mutation, the result is even more significantly effective. For some of the specific targeted drugs, a number of related studies found that they are having a promising future with positive research results. A latest study even stressed that it is more effective for patients with EGFR mutations to adopt the Third-Generation EGFR-TKI at the frontline. Apart from that, the ALK fusion gene-positive of the third generation drugs in lung cancer was also available on the market. For other target mutations that are rarely seen, the clinical trials of corresponding drugs are still making progress. It is believed that lung cancer will be eventually treated as the real meaning of chronic diseases to cure, benefiting more patients.

\section{Dialogue}

AME: We know that you were involved in a number of national, Asian and worldwide multicenter clinical trials. During those clinical trials, which one impressed you the most? What are the names of the joining institutions? What have you learnt from it?

Prof. Jian: I had a rather profound impression on two clinical trials. The first trial was from 1987 to 1988, when I just graduated from the university and joined a so-called "Vienna Program", which is a worldwide clinical study of non-small cell lung cancer. At that time, the research management was relatively rough. The second trial was a so-called "Interest Research", a study of gefitinib versus docetaxel. It was also the very first study of targeted therapy in China. This study was carried out by a strict operative system, which gave feedback to researchers and patients timely on any side effects of gefitinib and the related test results. In the middle of the trial, we received a negative result from a clinical trial of gefitinib comparing with placebo. After we had informed the ethics committee and the patients, we asked patients to sign for the consent once again, as it was the only way the study could be continued and we regarded it as a success. For China, clinical research has improved the overall level of medical care. For doctors, it cultivates them through a rigorous enforcement in scientific research, and enhances the level of data management and statistical analysis. In addition, it also provides them with a lot of opportunities to exchange ideas with the peers who are from others institutions, including Shanghai Cancer Hospital, Peking Union Medical College Hospital, Shandong Cancer Hospital and so on. To patients, it gives them a chance to contact with the world's latest treatment.

\section{AME: Can you share an impressive case with us?}

Prof. Jian: I was most impressed by a patient with nonsmall cell lung cancer, who participated in a trial of Iressa's expanding study in China. After prescribing him with Iressa, his lesions were completely absorbed and it lasted for 13 years. During the years of receiving medication, his family suffered from a great loss of his daughter. At that time, he took antidepressants while insisting on receiving the treatment simultaneously. When the tumors in him were growing bigger that he was not able to walk, Prof. Jian suggested him to receive a lung puncture to detect for new genes. After three-times of performing lung puncture on him, the result of T790 is finally showed positive. After he took 9,291 drugs, the lesions were significantly reduced again and he can even walk for now. During the onset of illness, this man was not yet 60 years old, and he is more than 70 years old now, we could tell that the patient has a very strong desire to live. In view of this, I think patients and doctors should have the faith in each other and cooperate with each other, and find the best means of treatment together.

\section{AME: Do you have any messages to young doctors in the department?}

Prof. Jian: I think the work of young doctors now is rather demanding. They have to do a lot of clinical scientific research and write articles continuously at the same time in order to grab the chance to get promoted. However, as a doctor, when it comes back to the basics of medicine, we focus on clinical practices and accumulate our experiences to do better. Being equipped with surgical skills, yet a lack of clinical practices is literally like an air loft, which contains zero real value at all. Therefore, I suggested that young doctors should open their eyes and spend more time on clinical practices to broaden their horizons, as patients 


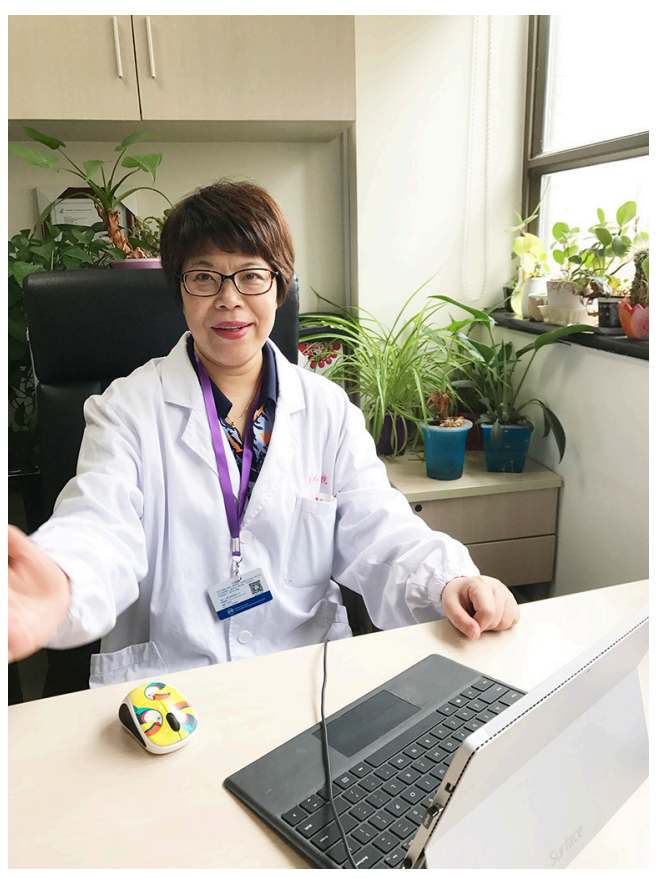

Figure 2 Prof. Jian's daily routine.

themselves are indeed our best textbooks.

\section{AME: How do you usually relieve the pressure and fatigue of your work?}

Prof. Jian: I used to feel I was not under too much work pressure as I love my work very much. Recently, I felt like I am getting older and my working intensity is getting bigger, I would feel tired sometimes. I usually choose to gather with my friends, having a tea and chatting with them. When I am alone, I will choose to go for a leisure reading, listen to music, watch a movie, attend painting exhibitions, and so forth. They help with soothing my emotions.

\section{AME: What are the values do you think a qualified doctor should have?}

Prof. Jian: For me, the most important thing is that you ought to love your career of being a doctor, treating it as a pursuit of life, and as a fun of life. Apart from that, you ought to give the best of you for being a doctor by always taking an initiative to study cases or some scientific research, and doing your utmost to find the best means of treatment for the patients in order to prolong their lives (Figure 2). From saving patents' lives, you will obtain the sense of accomplishment and happiness through the accumulation of medical knowledge and experience.

\section{AME: Did you ever regret choosing to be a doctor?}

Prof. Jian: I do not regret it. Indeed, I quite enjoy being a doctor by profession. I always keep thinking on just one single case, until I found the best treatment for my patients. For instance, I met a patient today who is with a tumor, suffering from a lower limb paralysis and incontinence. Under most circumstances, doctors may first diagnose him with a tumor's bone metastases, yet this patient had symptoms of fever two days before. Therefore, I suspected that he might happen to have a multiple neuritis, which is caused by other infectious diseases of the nervous system. Therefore, we have been constantly thinking, excluding as many as possible to look for solutions, and be more considerate for the patients. "I enjoyed working out the reasoning process of a diagnosis like a detective," said Prof. Jian. Furthermore, oncologists can rely on their own experience and knowledge upon a diagnosis, so that they can be their patient's spiritual dependence and be their accompanies until the end of their lives, which shall made them most proud of their work as a doctor.

\section{AME: If you are not a doctor, what else will you be interested?}

Prof. Jian: I will probably choose a career that is rather professional and challenging! For example, engineers and so on.

\section{Acknowledgements}

None.

\section{Footnote}

Conflicts of Interest: The authors have no conflicts of interest to declare.

(Editors: Crystal Yan, Monica Liu, Vicky Wong, AME Publishing Company, jtd@amepc.org)

Cite this article as: Yan C, Liu M, Wong V. Prof. Hong Jian: dreams come true-stay true to yourself! J Thorac Dis 2017;9(Suppl 11):S1176-S1180. doi: 10.21037/jtd.2017.09.136 